PAPER

\title{
Longlasting antalgic effects of daily sessions of repetitive transcranial magnetic stimulation in central and peripheral neuropathic pain
}

\author{
E M Khedr, H Kotb, N F Kamel, M A Ahmed, R Sadek, J C Rothwell
}

See Editorial Commentary, p 761

J Neurol Neurosurg Psychiatry 2005;76:833-838. doi: 10.1136/jnnp.2004.055806

See end of article for authors' affiliations

Correspondence to

Dr E M Khedr, Department of Neurology, Assiut University Hospital, Assiut, Egypt; Emankhedr99@ yahoo.com

Received 7 October 2004 Revised version received 29 November 2004

Accepted2December 2004
Background and objective: A single session of repetitive transcranial magnetic stimulation (rTMS) over motor cortex had been reported to produce short term relief of some types of chronic pain. The present study investigated whether five consecutive days of rTMS would lead to longer lasting pain relief in unilateral chronic intractable neuropathic pain.

Patients and methods: Forty eight patients with therapy resistant chronic unilateral pain syndromes (24 each with trigeminal neuralgia (TGN) and post-stroke pain syndrome (PSP)) participated. Fourteen from each group received 10 minutes real rTMS over the hand area of motor cortex $(20 \mathrm{~Hz}, 10 \times 10 \mathrm{~s}$ trains, intensity $80 \%$ of motor threshold) every day for five consecutive days. The remaining patients received sham stimulation. Pain was assessed using a visual analogue scale (VAS) and the Leeds assessment of neuropathic symptoms and signs (LANSS) scale, before, after the first, fourth, and fifth sessions, and two weeks after the last session.

Results: No significant differences were found in basal pain ratings between patients receiving real-and sham-rTMS. However, a two factor ANOVA revealed a significant " \pm TMS" $\times$ "time" interaction indicating that real and sham rTMS had different effects on the VAS and LANSS scales. Post hoc testing showed that in both groups of patients, real-rTMS led to a greater improvement in scales than sham-rTMS, evident even two weeks after the end of the treatment. No patient experienced adverse effects.

Conclusion: These results confirm that five daily sessions of rTMS over motor cortex can produce longlasting pain relief in patients with TGN or PSP.
S timulation of the motor cortex for the treatment of certain forms of refractory neurogenic pain has attracted much interest in recent years. Tsubokawa et al ${ }^{1}$ first showed that central post-stroke pain could be reduced by means of chronic motor cortex stimulation (MCS) through implanted epidural electrodes. Further studies proved that MCS could also relieve trigeminal neuropathic pain and central pain in Wallenberg's syndrome. ${ }^{23}$ However, such treatments are invasive and the outcome varies from patient to patient. A number of studies have shown that a single session of repetitive transcranial magnetic stimulation (rTMS) can relieve pain transiently in some patients with chronic neuropathic pain, ${ }^{4-6}$ although others have found the effect to be small and not significant. ${ }^{7}$

The question we address here is whether the duration of pain relief can be extended by repeated application of rTMS every day for five days. Lefaucheur et $a l^{8}$ reported that pain was well controlled in a single patient with drug resistant neuropathic pain for 16 months by monthly sessions of motor cortex rTMS. In contrast, Topper et a ${ }^{9}$ failed to see any long term therapeutic effect of three weeks' daily parietal cortex rTMS in two patients with pain due to longstanding unilateral avulsion of the lower cervical roots even though pain was reduced for 10 minutes in the same individuals after a single session of rTMS. We have extended these studies to a much larger group of patients using rTMS over the conventional motor cortex site.

\section{PATIENTS AND METHODS}

\section{Patients}

We conducted this study at the Department of Neurology, with participation of the Chronic Pain Unit, at Assiut University Hospital, Assiut, Egypt, between March 2003 and
March 2004. The study included 48 patients with unilateral chronic neuropathic pain; 24 had trigeminal neuralgia (TGN) as a peripheral neuropathic pain, and 24 had post-stroke pain (PSP) as a central type of neuropathic pain. All patients had been treated with various medications, including anticonvulsants, narcotic or non-narcotic analgesics and antidepressants, without satisfactory pain control. Three of the patients with TGN had persistent pain even after microvascular decompression.

Clinically, the diagnosis of TGN was based on the criteria of the International Association for the Study of Pain..$^{10}$ Sixteen women and eight men (mean (SD) age 51.5 (10.7) years) had TGN with mean duration of illness of 39 (31) months. Pain was commonly evoked by trivial stimuli, including washing, shaving, smoking, talking, and brushing the teeth, but could also occur spontaneously.

The clinical diagnosis of PSP was based on history of cerebrovascular stroke (haemorrhagic or thromboembolic). Ten women and 14 men (mean age 52.3 (10.3) years) had PSP with mean duration of illness of 18 (17) months. All patients with PSP had minor motor deficits. Twelve had thalamic infarction, six had thalamic haemorrhage, four had lateral medullary syndrome, and two had parietal infarction as documented by CT scan of the brain. The patients complained of a spontaneous, abnormally painful sensation of great intensity that they described as burning, tearing, or deep-boring, mostly in the face and upper limb and trunk

Abbreviations: $A D M$, abductor digiti minimi; $C T$, computed tomography; LANSS, Leeds assessment of neuropathic symptoms and signs; MCS, motor cortex stimulation; PSP, post-stroke pain; RMT, resting motor threshold; rTMS, repetitive transcranial magnetic stimulation; TGN, trigeminal neuralgia; VAS, visual analogue scale 
area (spontaneous painful dysaesthesia). Neurological examination revealed an increased threshold for pinprick and thermal sensation in the painful area in all patients and a decrease in tactile and/or vibration sensations of varying degrees in some patients. All patients had minor motor deficit.

We excluded patients with intracranial metallic devices or with pacemakers or any other device. We also excluded those with extensive myocardial ischaemia and those known to have epilepsy. All patients participated in the study after giving written informed consent and the local ethical committee of Assiut University Hospital approved the experimental procedure.

The baseline assessment consisted of a full history and neurological examination followed by instruction in the use of a visual analogue scale (VAS). Each patient then provided two VAS ratings, and the mean was taken. After this the patients were assessed by the examiner using the Leeds assessment of neuropathic symptoms and signs (LANSS) pain scale, which is based on analysis of sensory description and bedside examination of sensory dysfunction. ${ }^{11}$ Measures of VAS and LANSS were taken at each follow up point.

Patients were randomly assigned to one of the two groups, depending on the day of the week on which they were recruited. One group (consisting of patients recruited on Saturday to Monday) received real-rTMS and the other group (recruited on Tuesday to Thursday) received sham-rTMS.

\section{Preparation}

The patient sat in a comfortable chair and was asked to relax as much as possible. Electromyography (EMG) recordings from the contralateral abductor digiti minimi (ADM) muscle were acquired with silver-silver chloride surface electrodes, using a muscle belly-tendon set-up, with a $3 \mathrm{~cm}$ diameter circular ground electrode placed on the wrist. A Dantec Keypoint electromyograph was used to collect the signal (Dantec, Skovlunde, Denmark). EMG parameters included a bandpass of $20-1000 \mathrm{~Hz}$ and a recording time window of $200 \mathrm{~ms}$. TMS was performed with a commercially available $90 \mathrm{~mm}$ figure of eight coil connected to Mag-Lite r25 stimulator (Dantec Medical, Skovelund, Denmark).

\section{Determination of resting motor threshold}

First we determined the optimal scalp location from which TMS evoked motor potentials of greatest amplitude in the ADM. We used a constant suprathreshold stimulus intensity and moved the figure of eight coil systematically in $1 \mathrm{~cm}$ steps to determine the scalp position from where TMS evoked motor potentials of maximum peak to peak amplitude in the target muscle. The coil was positioned tangentially to the scalp and oriented so that the induced electrical currents would flow approximately perpendicular to the central sulcus, at a $45^{\circ}$ angle from the mid-sagittal line. ${ }^{12}$ Single pulse TMS was then delivered to the optimal location starting at suprathreshold intensity and decreasing in steps of $2 \%$ of the stimulator output. Relaxation and EMG signals were monitored for $20 \mathrm{~ms}$ prior to stimulation. The resting motor threshold (RMT) was defined as the minimal intensity required to elicit motor evoked potentials of $50 \mu \mathrm{V}$ peak to peak amplitude in five out of 10 consecutive trials. ${ }^{13}$ The optimal scalp location and coil orientation was marked using a red marker to reuse for daily rTMS.

\section{Repetitive transcranial magnetic stimulation}

A few studies have investigated the best parameters to use for rTMS mediated relief of pain. Epidural stimulation usually employs submotor threshold pulses at $\sim 40 \mathrm{~Hz}$. All previous rTMS studies have used subthreshold intensities, usually set at $80 \%$ RMT. However, different groups have used different frequencies. Lefaucheur et $a l^{4}$ found $10 \mathrm{~Hz}$ to be more effective than $0.5 \mathrm{~Hz}$. In contrast, Rollnik et al used $20 \mathrm{~Hz}$. In a preliminary investigation we examined whether $20 \mathrm{~Hz}$ rTMS may be more effective than $10 \mathrm{~Hz}$ (both at $80 \%$ RMT) over a non-blinded two day treatment session. Since $20 \mathrm{~Hz}$ seemed marginally more effective, we elected to use $20 \mathrm{~Hz}$, $80 \%$ RMT stimulation in the main study.

Real-rTMS involved applying a train of rTMS once per minute for 10 minutes. Each train consisted of 200 pulses at $20 \mathrm{~Hz}$ and 80\% RMT (total duration of $10 \mathrm{~s}$ ) applied through a figure of eight coil over the identified motor cortical area corresponding to the hand of the painful side. The treatment was repeated every day for five consecutive days. Sham-rTMS was applied using the same parameters but with the coil elevated and angled away from the head to reproduce some of the subjective sensation of rTMS and yet avoid induction of current in the brain. ${ }^{14}$ However, since none of the patients had experienced rTMS previously they were unaware of which stimulation was real and which was sham. During the rTMS, all patients wore earplugs to protect the ears from the acoustic artefact associated with the discharge of the stimulation coil.

\section{Follow up}

Patients were followed up after the first, fourth, and fifth rTMS session, and 15 days after the last session, using the VAS and LANSS scales. The second author evaluated these measures blindly-that is, without knowing the type of rTMS.

\section{Data analysis}

Pain level was assessed at baseline, after the first, fourth, and fifth rTMS session, and 15 days after the last session using the VAS and LANSS scales. Values for both patient groups (TGN and PSP) and each rating scale (VAS and LANSS) were analysed in separate two factor analysis of variance (ANOVA) with "time after start of treatment" and " \pm rTMS" as main factors. The Greenhouse-Geisser correction of degrees of freedom was used when necessary to correct non-sphericity of data. The percentage modification of the pain level was calculated from the scores measured before and after the rTMS sessions, both real and sham, by the following equation:

\section{(post-rTMS - pre-rTMS pain scores) $\times 100 /$ pre-rTMS pain score}

for VAS and LANSS. Individual effects of rTMS were also classified into three categories:

- good-reduction of pain score by $\geqslant 70 \%$

- satisfactory-reduction of pain score by $\geqslant 40-69 \%$

- poor-reduction of pain score by $<40 \%{ }^{15}$

\section{RESULTS}

There was no significant difference between the VAS and LANSS scores of the real and sham groups at baseline. However, fig 1 shows that the scores of the patients who received real-rTMS decreased more over the course of the treatment than those who received sham-TMS. This was confirmed in a two factor repeated measures ANOVA separately in each group of patients with "time of assessment" and " \pm rTMS" as main factors. In both the TGN and PSP groups, there was a significant interaction between "time" and " \pm rTMS" for both the VAS and LANSS scores $\left(\right.$ TGN VAS $F_{1.5,32.1}=4.7, \mathrm{p}=0.025 ;$ TGN LANSS $F_{1.8,39}=11.3$, $\mathrm{p}<0.001$; PSP VAS $F_{1.6,34.4}=26.6, \mathrm{p}<0.001 ;$ PSP LANSS $\left.F_{1.3,28.8}=25.5, \mathrm{p}<0.001\right)$. Post hoc testing showed that there 

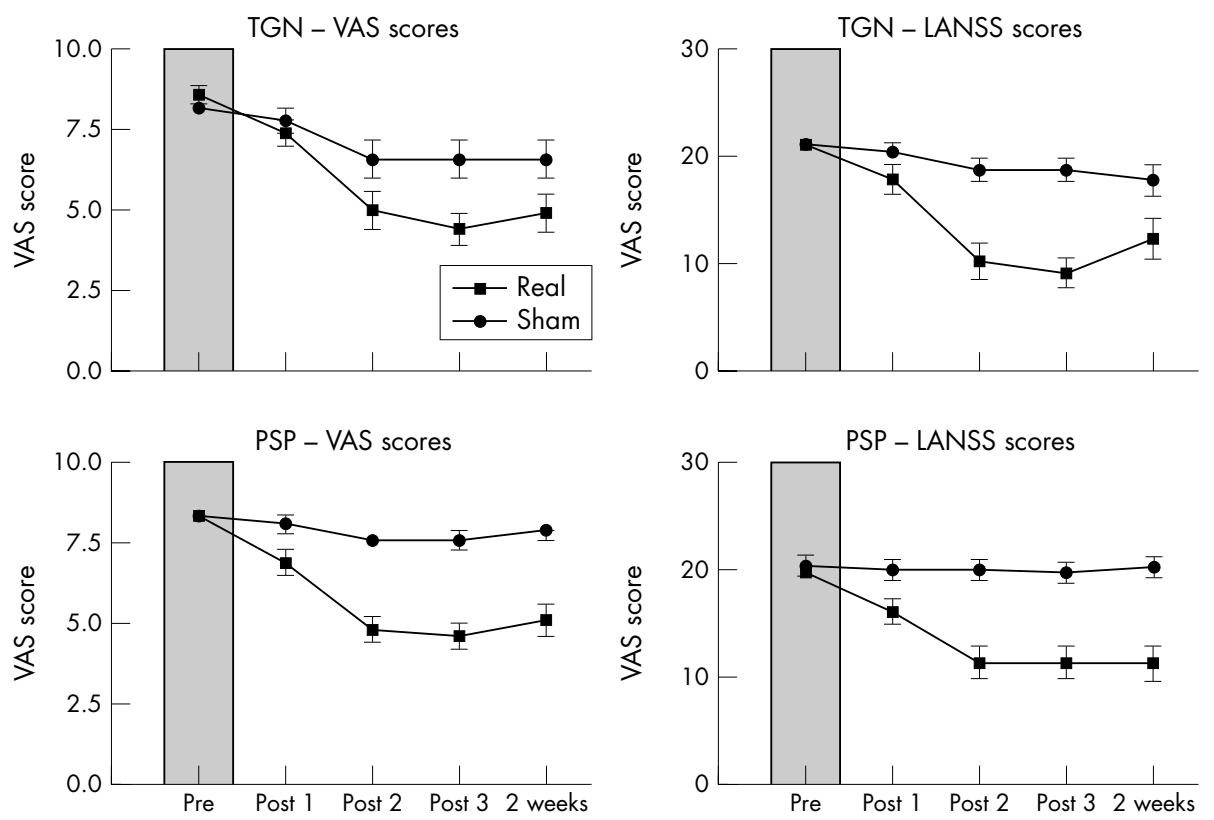

Figure 1 Changes in mean pain rating scores (visual analogue scale (VAS) and Leeds assessment of neuropathic symptoms and signs (LANSS) pain scale) at the five assessment points for the two groups of patients. The first assessment was immediately prior to commencing repetitive transcranial magnetic stimulation (rTMS) treatment (Pre), the second (Post 1) was immediately after the first session of rTMS, and then after the fourth (Post 2) and fifth (Post 3) rTMS sessions, and 15 days (2 weeks) after the last session. As the data show the mean scores of the patients who received real-rTMS decreased more over the course of the treatment than those who received sham-TMS. In both, the trigeminal neuralgia (TGN) and post-stroke pain (PSP) groups, there was a significant interaction between "time" and " \pm rTMS" for both the VAS and LANSS scores (see text for details). In both groups of patients improvement was greatest in those receiving real-TMS.
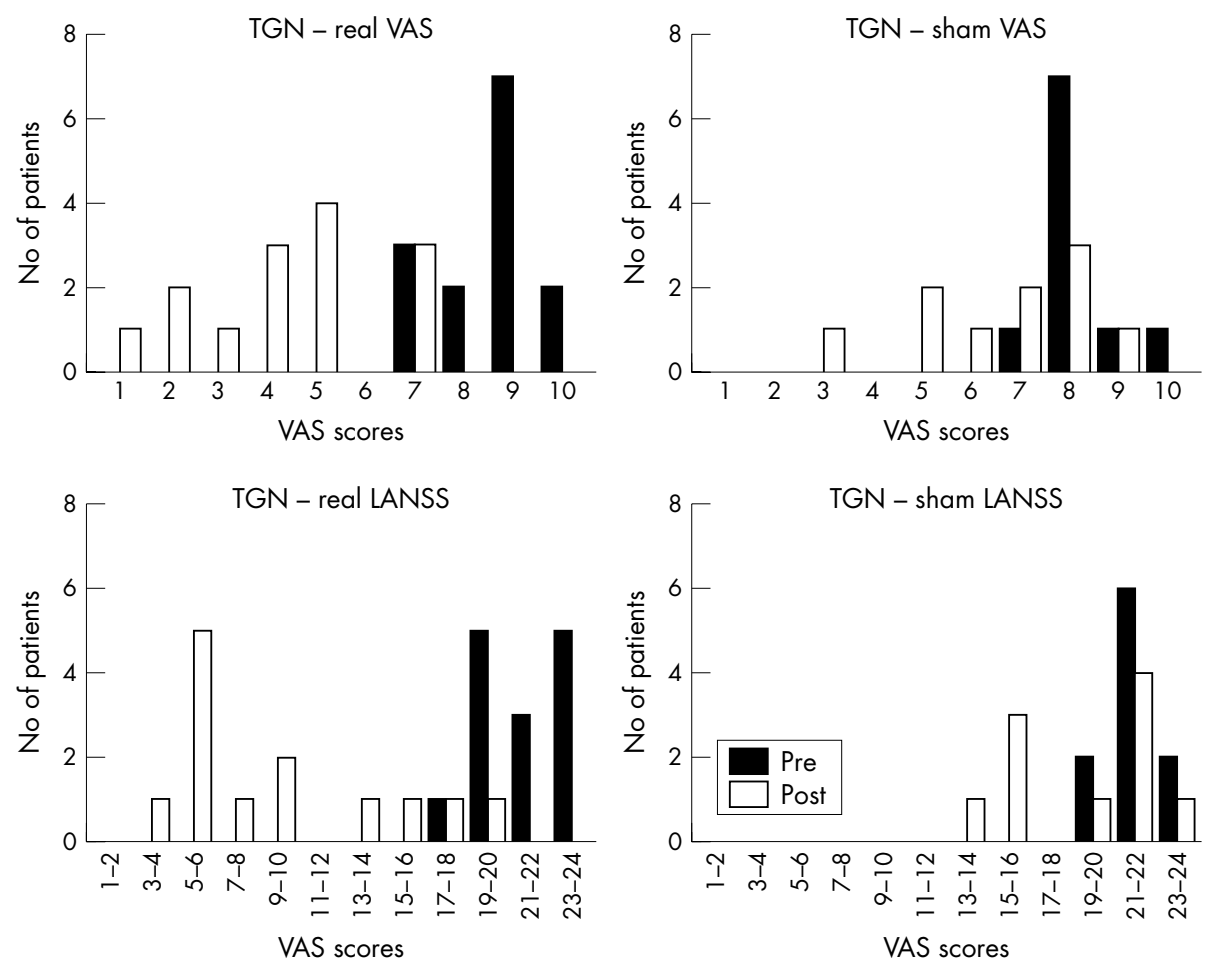

Figure 2 The distribution of rating scores among patients with trigeminal neuralgia (TGN). The graphs compare baseline data with that at the time of maximum effect immediately after the last treatment session. Although pain scores decreased after real repetitive transcranial magnetic stimulation (rTMS), the effect was greater in some individuals than others. So even after real-rTMS, a few patients were still in the baseline range. 

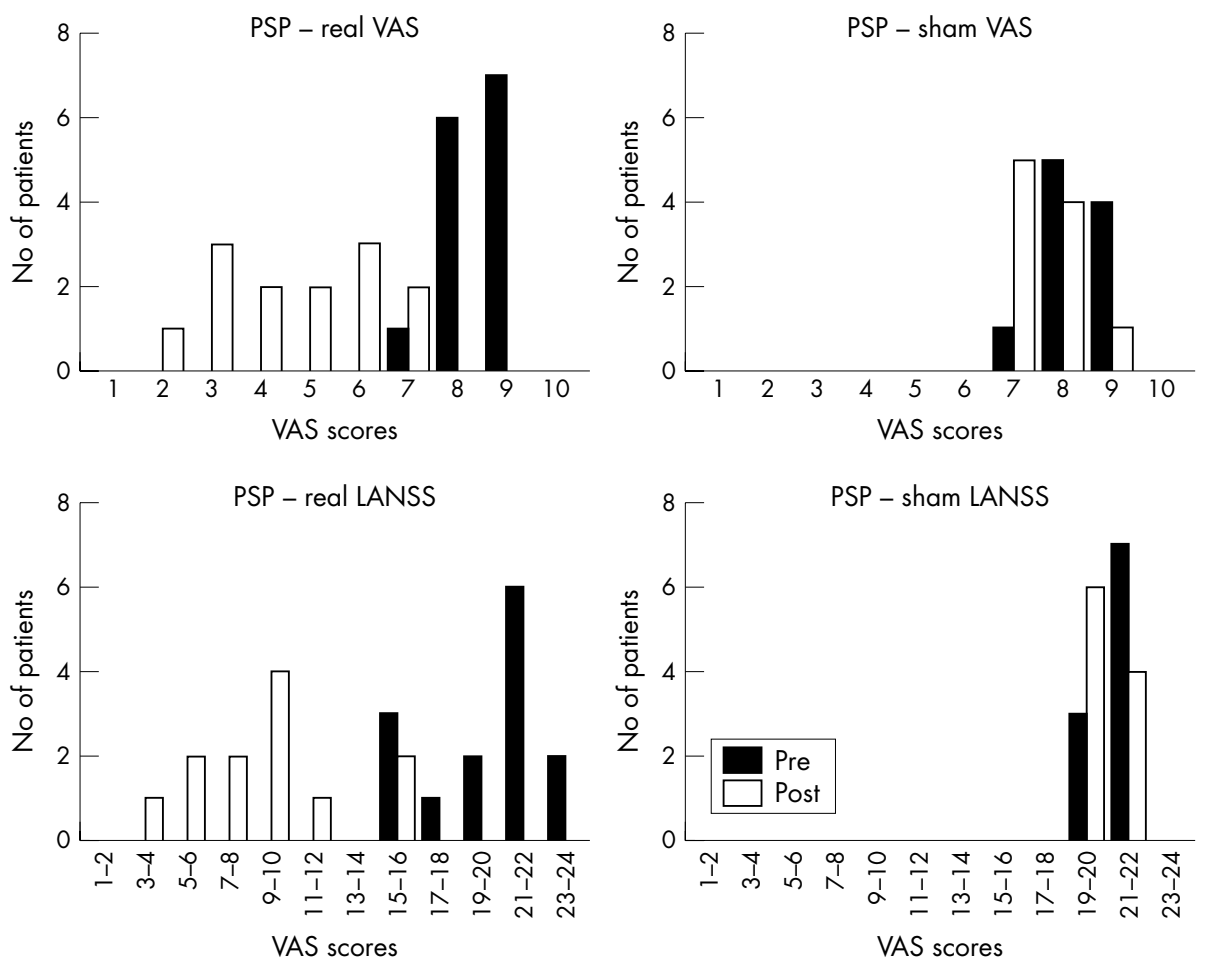

Figure 3 The distribution of rating scores among patients with post-stroke pain (PSP). The graphs compare baseline data with that at the time of maximum effect immediately after the last treatment session. Although pain scores decreased after real repetitive transcranial magnetic stimulation (rTMS), the effect was greater in some individuals than others. So even after real-rTMS, a few patients were still in the baseline range.

was a significant decrease in pain ratings at all time points after real rTMS compared with baseline (paired $t$ tests; $p<0.05$ ) in both groups of patients. There was no change in LANSS ratings after sham-rTMS in the PSP group although there was a small decrease in the patients' VAS scores after the fourth and fifth sessions and at two weeks' follow up. Both LANSS and VAS scores decreased in the TGN group after the fourth and fifth sessions and at two weeks' follow up.

Since there were no obvious differences in the results of patients with PSP and TGN, we grouped the data and took the mean of both rating scales to calculate the percentage reduction in pain ratings produced by rTMS. In the real-rTMS group pain decreased by $45 \%$ compared with baseline measures at the end of the fifth treatment session and was still reduced by $40 \%$ two weeks later. In contrast, pain ratings in the sham group declined only by $5 \%$ and $2 \%$, respectively $(\mathrm{p}<0.001$ comparing the percent reduction after real and sham treatments).

Figures 2 and 3 show how treatment changed the distribution of rating scores in the patients with TGN and PSP, respectively. Data at baseline are compared with that at the time of maximum effect immediately after the last treatment session. Real rTMS decreases the pain ratings, but it is also clear that the effect is greater in some individuals than others, with a small number of patients in each group remaining in the baseline range, even after real rTMS. Tables 1 and 2 summarise outcomes after the fifth treatment session and two weeks later.

\section{DISCUSSION}

Epidural electrical MCS has been reported to ameliorate symptoms in some patients with intractable chronic pain of central and peripheral origin. ${ }^{16}$ However, $~ 30 \%$ of operated patients fail to respond so that there is a need to develop predictive tools to select patients for treatment. ${ }^{18}{ }^{18}$ TMS is a relatively new technology that offers the possibility of testing whether patients will respond to direct cortical stimulation by measuring their response to a period of non-invasive cortical stimulation. Repetitive TMS appears to stimulate motor cortex in a way similar to that produced by epidural stimulation, ${ }^{19}$ and can transiently reduce pain in some groups of patients with neuropathic pain. ${ }^{4}$ The present data suggest that rTMS at $20 \mathrm{~Hz}$ given every day for five days can reduce pain ratings in patients with TGN and PSP for at least two weeks after the end of treatment. We conclude that repeated

Table 1 Individual effect on LANSS ratings of repetitive transcranial magnetic stimulation (rTMS) immediately after the last session and at two weeks' follow up. Values are $n$ (\%)

\begin{tabular}{llllllll}
\hline & \multicolumn{2}{l}{ After the fifth session } & & & \multicolumn{2}{l}{ Two weeks after the last session } \\
\cline { 2 - 3 } Subgroup & Poor & Satisfactory & Good & & Poor & Satisfactory & Good \\
\hline TGN real & $3(21.4)$ & $4(28.6)$ & $7(50)$ & & $7(50)$ & $4(28.6)$ & $3(21.4)$ \\
TGN sham & $8(80)$ & $2(20)$ & $0(0)$ & & $8(80)$ & $2(20)$ & $0(0)$ \\
PSP real & $4(28.6)$ & $8(57.2)$ & $2(14.3)$ & & $6(42.9)$ & $6(42.9)$ & $2(14.3)$ \\
PSP sham & $9(90)$ & $1(10)$ & $0(0)$ & & $9(90)$ & $1(10)$ & $0(0)$ \\
\hline
\end{tabular}

LANSS, Leeds assessment of neuropathic symptoms and signs; PSP, post-stroke pain; TGN, trigeminal neuralgia. 
Table 2 Individual effect on visual analogue scale (VAS) ratings of repetitive transcranial magnetic stimulation (rTMS) immediately after the last session and at two weeks' follow up. Values are $n(\%)$

\begin{tabular}{llllllll}
\hline & \multicolumn{3}{l}{ After the fifth session } & & & \multicolumn{2}{l}{ Two weeks after the last session } \\
\cline { 2 - 3 } Subgroup & Poor & Satisfactory & Good & & Poor & Satisfactory & Good \\
\hline TGN real & $4(28.6)$ & $7(50)$ & $3(21.4)$ & & $6(42.9)$ & $5(35.7)$ & $3(21.4)$ \\
TGN sham & $6(60)$ & $4(40)$ & $0(0)$ & & $8(80)$ & $2(20)$ & $0(0)$ \\
PSP real & $3(21.4)$ & $10(71.4)$ & $1(7.2)$ & & $5(35.7)$ & $7(50)$ & $2(14.3)$ \\
PSP sham & $9(90)$ & $1(10)$ & $0(0)$ & & $10(100)$ & $0(0)$ & $0(0)$ \\
\hline PSP, post-stroke pain; TGN, trigeminal neuralgia.
\end{tabular}

sessions of rTMS over the motor cortex may be, at least in some groups of patients, an effective way of providing relatively lasting relief of painful symptoms, and a screening procedure in others who might benefit from implantation of a chronic stimulating device.

There is a great deal of evidence that sessions of rTMS can lead to after effects on the excitability of the human cerebral cortex $^{14}$ and that repeated applications can prolong the duration of the effect even in healthy subjects. ${ }^{20}$ In view of these results, rTMS over the dorsolateral prefrontal cortex has been applied therapeutically with varying degrees of success to treat depressed and psychotic patients. ${ }^{21-23}$ In addition, rTMS of the motor cortex has been used to ameliorate dystonic symptoms ${ }^{24}$ and bradykinesia in Parkinson's disease..$^{25}$

Relatively few studies have investigated the effect of TMS in relief of chronic pain. Initial studies showed that a single session of rTMS over the motor cortex could lead to short term relief of pain ${ }^{4-6}$ in most cases. In the present study we tested whether repeated sessions of rTMS might prolong the control of pain. To our knowledge there are only two other previous studies on this topic. Topper et al ${ }^{9}$ reported no permanent reduction of pain in two patients with phantom limb pain-like syndrome after daily sessions of $10 \mathrm{~Hz}$ rTMS over the parietal cortex for three consecutive weeks. However, the pathophysiological basis of phantom limb pain-like syndrome may be different from other types of neuropathic pain. In addition, other successful studies of rTMS in pain relief have used the motor cortex as the target site rather than the parietal cortex. A second study of repeated rTMS in pain ${ }^{8}$ reported that neuropathic pain in a single patient was well controlled by monthly sessions of rTMS over the motor cortex at $10 \mathrm{~Hz}$. The present study used MCS in a daily regimen and found clear effects on pain ratings that outlasted treatment for up to two weeks.

A small placebo effect was found after the fourth treatment session in both groups of patients, particularly in the VAS scores. Lefaucheur et al found a similar sham effect, and indeed it would be unusual for there to be no placebo effect at all in a study such as this. However, the important point is that the effect of real-rTMS was much greater than that of the sham condition in both groups of patients and for both rating scales.

In the present study, the effects built up rather slowly, being only mild immediately after the initial session on the first day, but quite clear when tested immediately after the fourth session, and much greater than the placebo effect of sham stimulation. This is consistent with Lefaucheur et al's $\mathrm{s}^{4}$ original observation that pain relief after a single session was optimal two to four days after rTMS. Pleger et al $l^{6}$ recorded some pain relief 30 seconds after rTMS, but this intensified after 45 minutes. Since we assessed pain immediately after the first session, we may have missed the time of optimal response. However, by day 4, the effects were clear. Another explanation for the absence of significant pain relief after the first session could be related to the duration of the session in the present study, which was 10 minutes as compared with 20 minutes used in some of previous studies. ${ }^{4578}$

The mean degree of pain reduction $(\sim 45 \%$ in both rating scales) that we observed was both greater and longer lasting than has been reported in other studies. Although we cannot be certain of the reasons, it seems likely that this due to the combination of a larger number of rTMS pulses per session (2000 compared with 1000 of Lefaucheur et al ${ }^{4}$ or 400 of Topper et $a l^{9}$ ) plus the repeated sessions at daily intervals. Another explanation could be related to the site of pain. Most of our patients had either facial pain (TGN) or facial plus upper limb dysaesthesia (PSP). We applied rTMS over the hand area, which lies between the representation of the face and the arm. It might have been that stimulation here could have spread readily to both sites, as proposed by Lefaucheur et $a l^{5}$ and given a good overall reduction in pain ratings. Interestingly, there was a range in the response of individual patients: from excellent to virtually nothing. Precisely why this should occur is unknown, but since a similar range of responsiveness is seen after chronic cortical stimulation it was not entirely unexpected. One possibility is that the mechanism of pain relief relies on activation of corticothalamic projections ${ }^{9}$ that may be damaged in some of the patients. Nevertheless, the fact that rTMS can probe the range of interindividual effects makes it a good tool to screen patients for potential chronic implantation.

The mechanisms responsible for the effect of MCS on pain are still unknown. Several authors have observed an increase of cerebral blood flow in the ipsilateral thalamus, orbitofrontal and cingulate gyri, and in the upper brain stem during $\mathrm{MCS}^{16}$. As noted above it may be that activity in the projections to the thalamic nuclei from the motor and premotor cortices is modulated by rTMS entailing a cascade of synaptic events in pain related structures receiving afferents from these nuclei, including the medial thalamus, anterior cingulate, and upper brain stem.

Our results are compatible with previous work showing that direct electrical stimulation of motor cortex with implanted electrodes is an effective treatment in some patients for control of chronic deafferentation or neuropathic pain. ${ }^{26}{ }^{27}$ Here we show that repeated daily sessions of rTMS are able to mimic these effects in patients with TGN and PSP.

\section{Authors' affiliations}

E M Khedr, N F Kamel, M A Ahmed, R Sadek, Department of Neurology, Assiut University Hospital, Assiut, Egypt

H Kotb, Department of Anaesthesiology, Assiut University Hospital, Assiut, Egypt

J C Rothwell, Sobell Research Department of Motor Neuroscience and Movement Disorders, National Hospital for Neurology and

Neurosurgery, London, UK

Competing interests: none declared 


\section{REFERENCES}

1 Tsubokawa T, Katayama Y, Yamamoto T, et al. Chronic motor cortex stimulation in patients with thalamic pain. J Neurosurg 1993;78:393.

2 Meyerson BA, Lindblom U, Linderoth B, et al. Motor cortex stimulation as a treatment of trigeminal neuropathic pain. Acta Neurochir 1993;58:150-3.

3 Katayama Y, Tsubokawa T, Yamamoto T. Chronic motor cortex stimulation for central deafferentation pain: experience with bulbar pain secondary to Wallenberg syndrome. Stereotactic Funct Neurosurg 1994;62:295-9.

4 Lefaucheur JP, Drouot X, Keravel Y, et al. Pain relief induced by repetitive transcranial magnetic stimulation of precentral cortex. NeuroReport $2001 ; 12: 2963-5$

5 Lefaucheur JP, Drouot X, Menard-Lefaucheur I, et al. Neurogenic pain relief by repetitive transcranial magnetic stimulation depends on the origin and the site of pain. J Neurol Neurosurg Psychiatry 2004;75:612-16.

6 Pleger B, Janssen F, Schwenkreis P, et al. Repetitive transcranial magnetic stimulation of the motor cortex attenuates pain perception in complex regional pain syndrome type I. Neurosci Lett 2004;356:87-90.

7 Rollnik JD, Huber TJ, Mogk H, et al. Repetitive transcranial magnetic stimulation for the treatment of chronic pain-a pilot study. Eur Neurol 2002;48:6-10.

8 Lefaucheur JP, Drouot X, Menard-Lefaucheur I, et al. Neurogenic pain controlled for more than a year by monthly sessions of repetitive transcranial magnetic stimulation of the motor cortex. Neurophysiol Clin 2004;3:91-5.

9 Topper R, Foltys H, Meister IG, et al. Repetitive transcranial magnetic stimulation of the parietal cortex transiently ameliorates phantom limb painlike syndrome. Clinl Neurophysiol 2003;114:1521-30.

10 Merskey H, Bogouk N. Classification of chronic pain. Descriptions of chronic pain syndromes and definitions of pain terms. Seattle: IASP Press, 1994:59-71.

11 Bennett $M$. The LANSS Pain Scale: the Leeds assessment of neuropathic symptoms and signs. Pain 2001;92(1-2): 147-57.

12 Brasil-Neto JP, Cohen LG, Pascual-Leone A, et al. Rapid reversible modulation of human motor outputs after transient deafferentation of forearm: a study with transcranial magnetic stimulation. Neurology 1992;42:1302-6.

13 Rossini PM, Barker AT, Berardelli A, et al. Non-invasive electrical and magnetic stimulation of the brain, spinal cord and roots: basic principles and procedures for routine clinical application. Report of an IFCN committee. Electroencephalogr Clin Neurophysiol 1994;91:79-92.
14 Pascual-Leone A Tormos JM, Keenan J, et al Study and modulation of human cortical excitability with transcranial magnetic stimulation. J Clin Neurophysiol 1998;15:333-43

15 Nguyen JP, Lefaucher JP, Le Guerinel C, et al. Motor cortex stimulation in the treatment of central and neuropathic pian. Arch Med Res 2000;31:263-5.

16 Garcia-Larrea L, Peyron R, Mertens P, et al. Electrical stimulation of the motor cortex for pain control: a combined PET scan and electrophysiological study. Pain 1999;83:259-73.

17 Mertens $\mathbf{P}$, Nuti $C$, Sindou $M$, et al. Precentral cortex stimulation for the treatment of central neuropathic pain: results of a prospective study in a 20patient series. Stereotact Funct Neurosurg 1999;73:122-5.

18 Nguyen JP, Lefaucheur JP, Decq P, et al. Chronic motor cortex stimulation in the treatment of central and neuropathic pain. Correlations between clinical, electrophysiological and anatomical data. Pain 1999:82:245-51.

19 Di Lazzaro V, Oliviero A, Pilato F, et al. Comparison of descending volleys evoked by transcranial and epidural motor cortex stimulation in a conscious patient with bulbar pain. Clinical Neurophysiology 2004; 115:834-8.

20 Baron JC, Black SE, Butler AJ, et al. Neuroimaging in stroke recovery: a position paper from the first International Workshop on neuroimaging and stroke recovery. Cerebrovasc Dis 2004;18:260-7.

21 Geller V, Grisaru N, Abarbanel JM, et al. Slow magnetic stimulation of prefrontal cortex in depression and schizophrenia. Prog Neuropsychopharmacol Biol Psychiatry 1997;21:105-10.

22 George MS, Wassermann EM, Kimbrell TA, et al. Mood improvement following daily prefrontal repetitive transcranial magnetic stimulation in patients with depression : A placebo-controlled cross-over trial. Am J Psychiatry 1997; 12:1752-6.

23 Rollnik JD, Huber TJ, Mogk $\mathrm{H}$, et al. High frequency repetitive transcranial magnetic stimulation (rTMS) of the dorsolateral prefrontal cortex in schizophrenic patients. NeuroReport 2000;11:4013-15.

24 Siebner HR, Tormos JM, Ceballos-Baumann AO, et al. Low frequency repetitive transcranial magnetic stimulation of the motor cortex in writer's cramp. Neurology 1999:52:529-37.

25 Khedr EM, Farweez HM, Hassan I. Therapeutic effect of repetitive transcranial magnetic stimulation on motor function in Parkinson's disease patients. Eur J Neurol 2003; 10:567-72

26 Saitoh Y, Shibata M, Hirano SI, et al. Motor cortical stimulation for central and peripheral deafferentation pain. J Neurosurg 2000;92:150-5.

27 Carroll D, Joint C, Maartens N, et al. Motor cortex stimulation for chronic neuropathic pain: a preliminary study of 10 cases. Pain 2000;84:431-7.

\section{$\mathrm{ECHO}$}

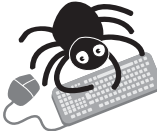

Please visit the Journal of Neurology, Neurosurgery, and Psychiatry website [www. jnnp.com] for a link to the full text of this article.
Association between hormone replacement therapy and subsequent stroke: a metaanalysis

Philip M W Bath, Laura J Gray

Objectives: To review completed trials assessing effect of hormone replacement therapy on subsequent risk of stroke, assessing stroke by pathological type, severity, and outcome.

Design: Systematic review of randomised controlled trials identified from the Cochrane Library, Embase, and Medline; reviews; and reference lists of relevant papers.

Studies reviewed: 28 trials, with 39769 subjects, were identified.

Review measures: Rates for cerebrovascular events analysed with a random effects model. Sensitivity analyses for heterogeneity included phase of prevention (primary or secondary), type of hormone replacement therapy (oestrogen alone or combined with progesterone), type of oestrogen (estradiol or conjugated equine oestrogen), size of trial $(<5000$ or $>5000$ patients), length of follow up ( $\leqslant 3$ years or $>3$ years), sex (women only or men only), and trial quality (high or low).

Results: Hormone replacement therapy was associated with significant increases in total stroke (odds ratio 1.29 (95\% confidence interval 1.13 to 1.47 ), $\mathrm{n}=28$ ), non-fatal stroke ( 1.23 (1.06 to 1.44$), \mathrm{n}=21$ ), stroke leading to death or disability (1.56 (1.11 to 2.20), $\mathrm{n}=14$ ), ischaemic stroke ( 1.29 ( 1.06 to 1.56$), \mathrm{n}=16)$, and a trend to more fatal stroke (1.28 (0.87 to $1.88), \mathrm{n}=22$ ). It was not associated with haemorrhagic stroke ( $1.07(0.65$ to 1.75$), \mathrm{n}=17$ ) or transient ischaemic attack (1.02 (0.78 to 1.34$), \mathrm{n}=22)$. Statistical heterogeneity was not present in any analysis.

Conclusions: Hormone replacement therapy was associated with an increased risk of stroke, particularly of ischaemic type. Among subjects who had a stroke, those taking hormone replacement therapy seemed to have a worse outcome. Hormone replacement therapy cannot be recommended for the primary or secondary prevention of stroke.

\ BMJ 2005;330:342-345. 